\title{
Dynamics of fuel jet brightness areas in video shooting
}

\author{
Alexander Eskov ${ }^{1, *}$, Anatoliy Sagalakov ${ }^{2}$, Sergey Kulmanakov ${ }^{2}$ and Ivan Kirushin ${ }^{2}$ \\ ${ }^{1}$ Krasnodar University of the Ministry of the Interior of Russia, 128, Yaroslavskaia St., 350005 \\ Krasnodar, Russia \\ ${ }^{2}$ Altai State Technical University, 46, Lenin St., 656099 Barnaul, Russia
}

\begin{abstract}
Applying of pixels separation brightness threshold is usually used to find the object boundaries. It is proposed to use a method for dividing the brightness of pixels to assign them to one of the zones of the fuel jet. A low brightness area with higher concentration of fuel, and vice versa, a zone with sufficient brightness (except the background luminance) has a lower concentration. Studying the dynamics of the change in brightness in the zones of a jet, we can talk about the quality of fuel atomization nozzles that can be different under different conditions.
\end{abstract}

\section{Introduction}

During the last decades requirements for increasing power, efficiency and decreasing the amount of exhaust emissions in modern internal-combustion engines are continuously raising. It is important to point that even a small improvement in characteristics of engines will cause a big change in efficiency and ecology overall.

The most important system in the internal combustion engine work is a fuel-supply system, in which the process of producing air-to-fuel depends on manufacturing accuracy of separate parts and its degree of wear condition during exploitations. In particular, the quality of creating sprayers can be controlled according to the parameter of fuel atomization. Among existing indirect methods of controlling the structure of fuel jets the most preferable are the optical methods. What depends on the laws of distribution and light propagation in the environment of impulse particulate matters of a diesel spray (or another liquid)? In this report a method, based on high-speed video registration of forming a spray with a further data-processing and calculations of concentration of fuel drops, is given.

The basic parameters, describing impulse particulate flow of sprayed liquid are: the average size of drops, its homogeneity and equitability of distribution concentration through the volume of a jet, the speed and direction of particle movements. The geometrical parameters are: the disclosing angle and the jet length. For practical needs the most important parameters are atomization brittleness, the concentration of fuel in the section of a jet and the jet length.

The aim of this research is to investigate methods of diagnostics of different parameters that influence the quality of sprayed liquid fuel in different conditions.

\footnotetext{
* Corresponding author: alesc72@mail.ru
} 


\section{Experimental setup of speed video stream of atomized fuel}

Speed video- photo-shooting of quickly changing processes of injections and fuel burning in the camera of internal-combustion engines is used successfully in some of scientific institutes and constructing departments of industrial organizations in many countries. In Polzunov Altai State Technical University the battery fuel system (electrical type Commonrail $\mathrm{CR}$ ) was designed. It allows to reach the fuel pressure in the nozzle to $180 \mathrm{MPa}$. CR system is installed on a single cylinder engine stand CC-2. To investigate the process of diesel fuel atomization the stand of a speed video stream of atomized fuel was developed. The diagram is shown in Fig. 1.

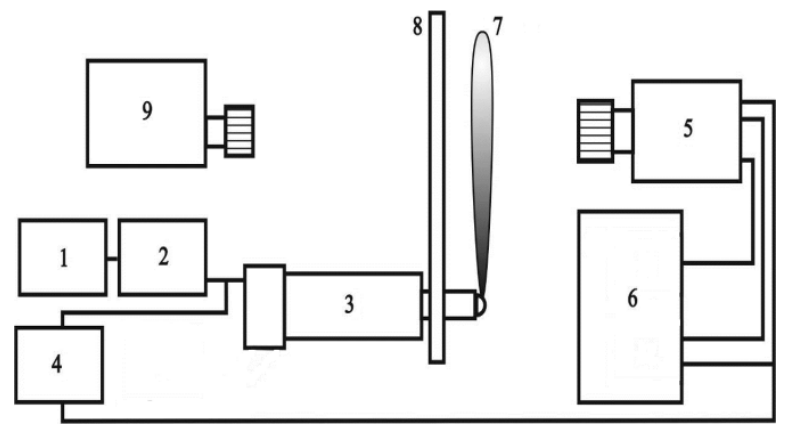

Fig. 1. Stand scheme of speed.

The control device of the laboratory setup CC-2 (1) takes the pulse to the control unit (2) opening the valve of a high-pressure nozzle (3). The pulse from the valve (2) is applied to the synchronization unit (4). After the launch of video camera work synchronizes, the leading edge of the pulse supplies to the injector. An information signal is transmitted from a camera (5) to a personal computer (6) by the cable of a controller camera. Thus, the moment signal camera exposure (5) is shown on the computer (6). Clock signals from the synchronization device (4) and the moment signal camera exposure (9) are necessary to determine the recording time of a specified frame in the movie, which is detected by the camera (5) and recorded on a personal computer (6).

Speed video camera "Videosprint" is $1.2 \mathrm{~m}$ from the screen (8) in the form of mattesurfaced glass. Behind it a permanent source of light (9) with incandescent 450 watt light is installed.

In the center of the screen there is a technical hole for the fuel nozzle sprayer. The used nozzle has four generated oppositely directed fuel jets (7), which work in the plane, in parallel to the screen so that one of them is located next to the screen glass(8) which is the one that was recorded. The other three jets are censored by special devices and the fuel in them instead of spraying in the air comes back in the place for fuel.

\section{Image processing fuel jets}

A computer is used as a device for processing and storage of received image. It contains specially created software «Jet Researcher». The fuel jet has unclear boundaries; therefore the method of identification of the boundaries of fuel is very important.

After analyzing the given messages there were identified groups of objects, which are interesting for the investigators. The methods, segmentations were used to find bright zones; therefore, we had to divide the images on the first plan and the second one. Segmentation allowed us to highlight the parts of image, which are to our mind homogeneous. For this the division of the image into parts with the same appearance was 
made. The universal method of segmentation, which could give a result fitting all the images, was not created. [2].

There were identified numbers which describe the boundary brightness of pixels that fit areas on the painted jet with which the comparing of each pixel was made. As a result, the pixel joins one of the groups as its brightness is higher and the boundary of the choice is usually made by using the diagram image. If the image consists of two areas one of which is dark and the other one contains light pixels then it can be said that the diagram will have two maximums.

Pixels with intensity lower than the threshold number are marked as the ones that belong to the object, the other ones belong to the background. Comparing is made by pixels with an image of a background on a recorded scene without a jet (pic. 2). the brightness of a sprayer in the given image has a low meaning (dark) and is located near the brightness of a jet. Therefore, in both methods of investigation of the image the first step is getting the image out of a video.

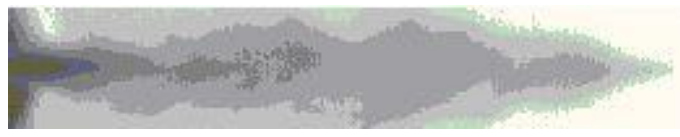

Fig. 2. Calculation of the jet boundaries by comparison with the background image.

The first value of a jet is located in the spot which fits the coordinates of the spout spray; the images are received with those settings: a frequency of 7042 frames / s, with an exposure of $136 \mathrm{~ms}$, the frame format is $1280 \mathrm{~h} 71$ pixels with $142 \mathrm{~ms}$ intervals.

\section{Dynamics of brightness zones of jet atomized fuel}

Fig. 3 shows the changes of the area of diesel fuel spray for different pressures. From the graphics it is possible to see that with an increase of pressure the surface of a jet with high pressure is more than the surface of a jet with smaller pressure. Jets with high pressure do not have only big length but also big width. The diagrams that show changes in surface have non-linear nature like the dependence of the jet length at the time of different pressures.

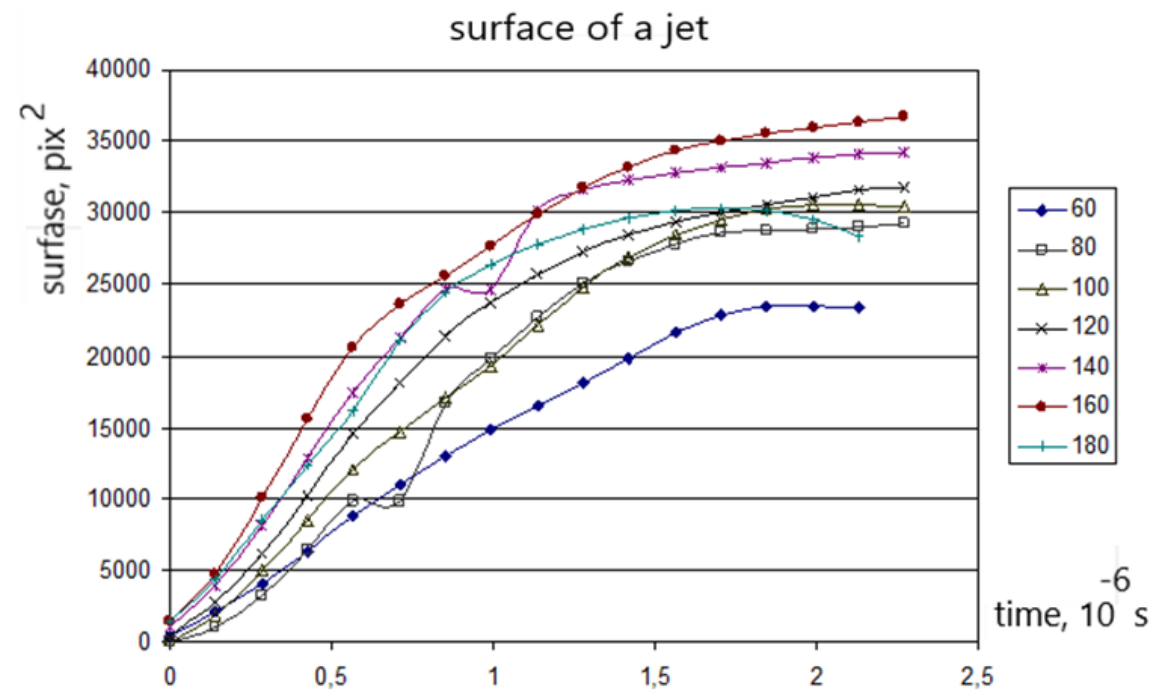

Fig. 3. Area of SPRAY diesel fuel without pilot injection. 
Each point was obtained by processing images of fuel jets on the developed program area calculation brightness zones [4, 5].The darkest gradation ADC (60 deg.) corresponds to a region with high fuel concentration usually in the middle of a jet and at the beginning of spraying, in the first shots, while the fuel jet has not started disintegrating yet. With a rise of pressure it is possible to see the growth of an amount of time needed for disintegration of a dark part from 1,2 to 1,4 ms. The bigger pressure of fuel forms a grand jet length, so the top location of a jet is formed further from the sprayer. This jet starts to disintegrate later. There is a trend offset of the maxima areas of gradation $(80,100,120,140$ deg.) at time upward. For the brightest area (160 deg) the maxima will probably appear later when the whole jet disintegrates and becomes homicidal in brightness with its subsequent reduction.

Fig. 5 shows the changes in the area of the jets sprayed fuel RME for different pressures. From the graphic it is possible to see that with an increase of the pressure of a sprayer at the moment the surface of a jet with a bigger pressure is bigger than the surface of a jet with a low pressure. Fuel consumption RME is equal to the consumption during the experiments with a diesel fuel.

regulated surface $(60 \mathrm{MPa})$
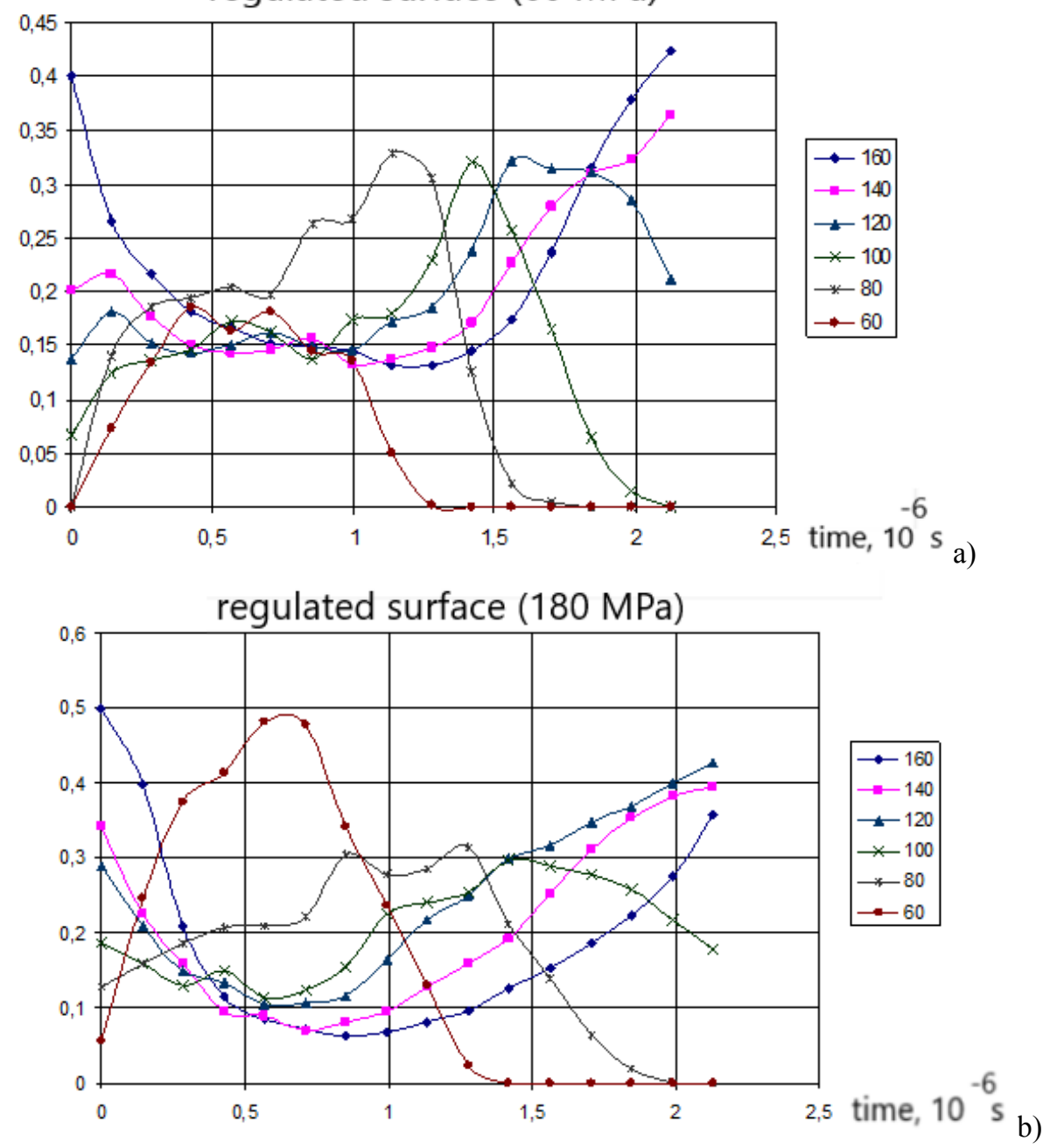

Fig. 4. Example of the dynamics of change of the normalized area of brightness zones of jet diesel fuel: a) for injection pressure of $60 \mathrm{MPa}, \mathrm{b}$ ) for injection pressure of $180 \mathrm{MPa}$. 


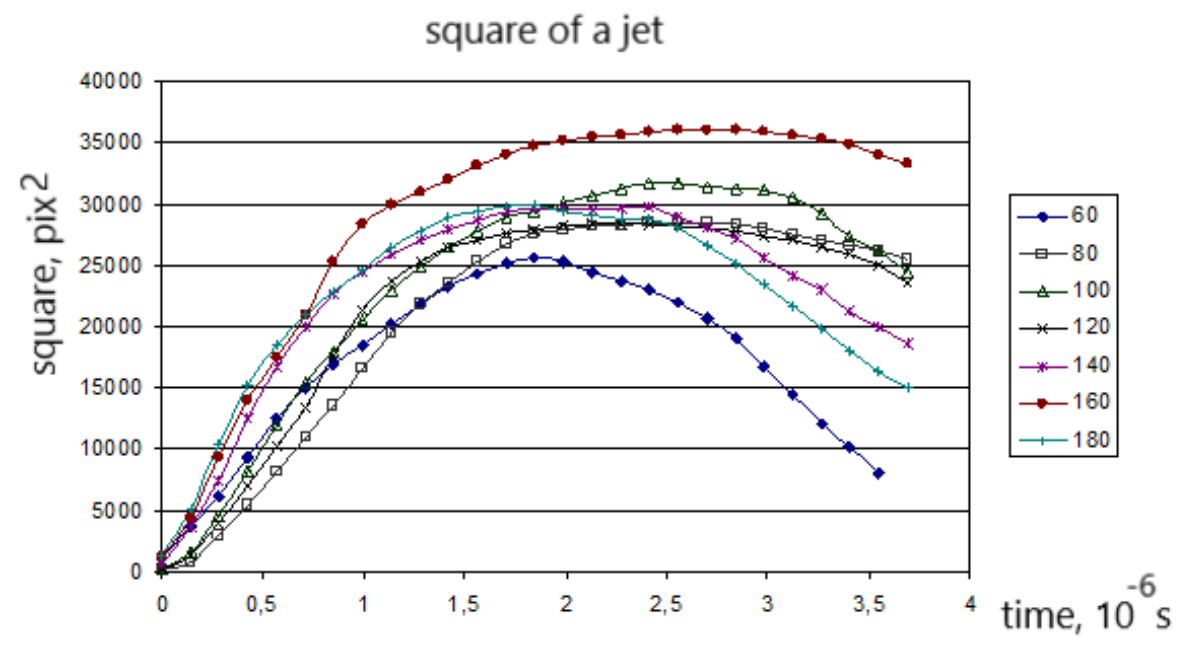

Fig. 5. Area of atomized jet diesel fuel with pilot injection portion.

\section{Conclusion}

The stand with high speed video shooting and the software demonstrates the comparison of the images of fuel jets with different fuel pressures and different types of fuel.

An increase of an area and a jet pressure comes with an increase of an injection.

The area of a jet with high concentration of fuel is located in the middle of a jet and in the beginning of an injection, while the fuel jet does not start to disintegrate. With an increasing pressure from 60 to $180 \mathrm{MPa}$, an increase of the time of the breakup of the dark area is of 1.2 to $1.4 \mathrm{~ms}$. The jet with a high pressure of an injection is longer and starts to disintegrate later.

By increasing the injection pressure, the area with dense fuel increases, the area of less dense zones reduces, conditional maximum of fuel areas move upward at time. Later, the dense area reduces; the fuel passes from more dense to less dense areas. In the end, the size of the brightest area increases.

\section{References}

1. The site of the company «Videoscan», http://www.videoscan.ru

2. M.A. Sojfer, Methods of computer image processing (2001)

3. A.V. Eskov, A.V. Maetskiy, A.M. Sagalakov, Optical method for research of process atomization of rape and diesel fuel, Sensors and Systems 5 (2013)

4. A.V. Eskov, A.V. Maetsky, Estimation of fuel jets on a program-technical complex of dispersion quality optical control, Internal combustion engines 2 (2012)

5. A.V. Eskov, A.M. Sagalakov, S.P. Kulmanakov, A.V. Maetskiy, I.I. Kirushin, Investigation of mixing processes in a diesel engine with the optical quality control of fuel atomization, Bulletin of the Altai science 1 (2014) 